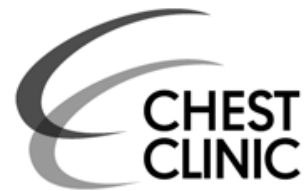
Royal Aberdeen Children's Hospital, Aberdeen, UK ${ }^{2}$ Department of Paediatric Respiratory Medicine and School of Medicine, University of Glasgow, Royal Hospital for Sick Children, Glasgow, UK ${ }^{3}$ Respiratory Unit, Freeman Hospital, Newcastle upon Tyne Tyne and Wear, UK ${ }^{4}$ Respiratory Unit, Aberdeen Royal Infirmary, Aberdeen, UK

\section{Correspondence to}

Dr Graham Douglas, Respiratory Unit, Aberdeen Royal Infirmary, Aberdeen AB25 2ZN, UK: graham.douglas@nhs.net

Received 18 March 2011 Accepted 8 June 2011 Published Online First 27 July 2011

\title{
British guidelines on the management of asthma: what's new for 2011?
}

\author{
Steve Turner, ${ }^{1}$ James Paton, ${ }^{2}$ Bernard Higgins, ${ }^{3}$ Graham Douglas ${ }^{4}$
}

Since 1999 the British Guidelines on the Management of Asthma have been produced jointly by the British Thoracic Society (BTS) and Scottish Intercollegiate Guideline Network (SIGN) using a rigorous evidence-based methodology. Sections within the guideline have been revised regularly, with the Pharmacological Management section being updated nearly every year. We believe this has resulted in a 'living guideline' that is responsive to new research and is current for clinicians. For 2011, two new sections have been added on Monitoring and Control and Asthma in Adolescents.

\section{MONITORING AND CONTROL}

There is increasing awareness that current control is a good predictor of future exacerbations. ${ }^{1}$ There is also some evidence that biomarkers might be useful in predicting future asthma control. ${ }^{2}$ Perhaps not unexpectedly, this new section is able to identify more areas where evidence is lacking than where evidence can be found to support practice. In adults and children there is very little evidence that addition of biomarkers to validated symptom scores improves asthma control; indeed, most of the evidence points towards biomarkers not assisting in the management of asthma. Such biomarkers include peak expiratory flow, spirometry, bronchial hyper-responsiveness and exhaled nitric oxide (FenO). Although not apparently helpful in routine monitoring, there is a little evidence that FenO might have a role in aiding stepping down and stopping inhaled corticosteroid treatment in children. The clinical utility of FenO has, however, yet to be adequately explored. The role of sputum eosinophilia to guide treatment is of proven benefit in adults (stepping up and stepping down) and children (stepping down only), although only in those with more severe asthma. Moreover, at present this test is only routinely available in a small number of tertiary centres. Good practice points include asking closed questions when assessing patients-for example, 'How many days per week do you use your blue inhaler?'-rather than open questions such as 'How is your asthma?' While the search for sensitive biomarkers for asthma control continues, titrating treatment against current control using a validated scoring system such as the Asthma Control Test remains the gold standard for monitoring care.

\section{ASTHMA IN ADOLESCENTS}

The WHO defines adolescence as between 10 and 19 years, a period that encompasses the transition through puberty to adulthood. It is estimated that there are 1.2 billion adolescents worldwide - that is, one in every five people in the world is an adolescent. For diagnosing and managing asthma in adolescents, the evidence base is limited. Recent research has focused on the prevalence of asthma and ecological risk associations rather than on diagnosis and management of asthma in adolescents.

Recent evidence from the International Study of Asthma and Allergy in Childhood (ISAAC) reported prevalence rates of severe asthma of around $11 \%$ in 13-14-year olds from English language centres. ${ }^{3}$ Perhaps surprisingly, the prevalence rates in adolescents are very similar to those in younger children. There is evidence of substantial underdiagnosis, estimated at between $20 \%$ and $30 \%$, even in those with severe symptoms. ${ }^{3} 4$ Identified risk factors for underdiagnosis include being female, smoking (active and passive), low physical activity and high body mass, family problems, race/ ethnicity and social disadvantage. Those with symptoms but without a diagnosis do not receive adequate healthcare and have substantial health consequences. ${ }^{5}$ It is because of such evidence that adolescents with asthma are often said to be 'lost in transition'. Clinicians seeing adolescents with any cardiorespiratory problems should therefore ask about symptoms of asthma.

Pharmaceutical trials often include adolescents, but there is usually no separate analysis by age. Recommendations for drug treatment are then extrapolated from younger or older subjects. Similarly, there is little age-specific guidance on inhaler devices for adolescents with asthma, although recent qualitative evidence has highlighted that many adolescents prescribed a metered dose inhaler with spacer do not use the spacer because they find it 'inconvenient'. 6 Adolescent preference for inhaler devices should therefore be considered as a factor in improving adherence with treatment.

One area little different from other areas of respiratory disease is the risk from smoking to adolescents with asthma. A recent study in Sweden highlighted that passive and active smoking are both significantly related to asthma and wheeze in adolescents. Maternal environmental tobacco smoke exposure was associated with lifetime symptoms but daily smoking in the adolescents was more strongly related to current wheeze (figure 1). ${ }^{7}$

If adolescents with asthma are hard to reach, there is very little evidence on where best to see them. Some innovative approaches have successively used schools as a setting for asthma education, including peer-led education. ${ }^{8}$ Australasian 


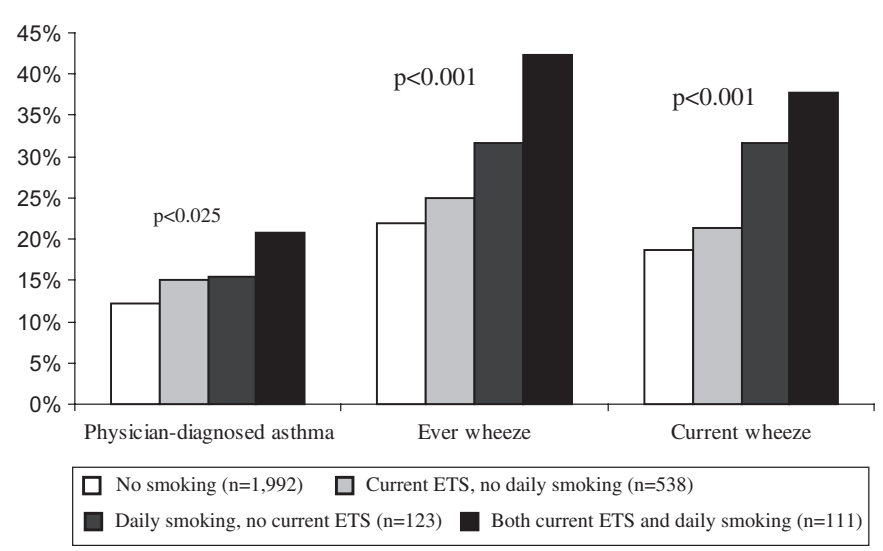

Figure 1 Prevalence (\%) of asthma and wheeze at age 16-17 years in relation to personal daily smoking and current maternal environmental tobacco smoke (ETS) exposure. ${ }^{7}$

countries have developed national programmes to promote asthma-friendly schools to ensure that schools can deliver emergency asthma care and to encourage students with asthma to take part in sport. School-based clinics may therefore improve attendance at asthma reviews.

The transition from paediatric to adult services is important for all adolescents with continuing asthma, irrespective of the severity. It is clear that adolescents need to be prepared to take responsibility for their own asthma management as well as to develop the necessary skills to negotiate health services effectively. Although there are no studies specifically about asthma, many studies have looked at the transition of adolescents with chronic illness and there are many published consensus recommendations. These emphasise that transition is a process over time rather than a single event such as moving to an adult clinic. The process should begin early and must involve the young person. Two points are particularly highlighted:

- Adolescents with asthma should be seen on their own, separate from their parents, for part of any consultation.

- The importance of discussing confidentiality and its limitations with the adolescent.

One area that practitioners must also remember is the need for guidance about work choices. While adolescents with mild asthma have been found to have only slightly more limitations in their working careers, they had a low awareness of occupations that might worsen asthma (such as exposure to dusts, fumes, sprays, exertion and temperature changes) and did not generally discuss these issues with their general practitioner. Future career choices should therefore be discussed with adolescents and occupations that might increase susceptibility to work-related symptoms highlighted.

The evidence informing these new areas is relatively poor, but the guideline is able to offer some recommendations for good clinical practice. Hopefully they will prove a stimulus to future research. Meanwhile, the process of updating these guidelines continues and, for 2012, there are plans to update sections on Pharmacological and Non-Pharmacological Management; Patient Education and Adherence; and Delivery of Care.

Acknowledgements We wish to acknowledge the enormous contribution made by those on the Evidence Review Groups and to Roberta James and Jan Manson at SIGN for providing literature searches, training in critical appraisal and supervision of the guideline process.

\section{Competing interests None.}

Contributors All four contributors are members of the Steering Committee of the British Guidelines on the Management of Asthma. BH and GD are co-chairs of the whole guideline while ST and JP are chairs of the Evidence Review Groups for each of these two new sections.

Provenance and peer review Not commissioned; externally peer reviewed.

\section{REFERENCES}

1. Taylor DR, Bateman ED, Boulet LP, et al. A new perspective on concepts of asthma severity and control. Eur Respir J 2008;32:545-54.

2. Taylor DR. Risk assessment in asthma and COPD: a potential role for biomarkers? Thorax 2009;64:261-4.

3. Lai CK, Beasley R, Crane J, et al. Global variation in the prevalence and severity of asthma symptoms: phase three of the International Study of Asthma and Allergies in Childhood (ISAAC). Thorax 2009;64:476-83.

4. Ayala GX, Yeatts K, Carpenter DM. Factors associated with asthma management self-efficacy among 7th and 8th grade students. J Pediatr Psychol 2009;34:862-8

5. Yeatts K, Johnston Davis K, Peden D, et al. Health consequences associated with frequent wheezing in adolescents without asthma diagnosis. Eur Respir $\mathrm{J}$ 2003;22:781-6.

6. Edgecombe K, Latter S, Peters $\mathrm{S}$, et al. Health experiences of adolescents with uncontrolled severe asthma. Arch Dis Child 2010;95:985-91.

7. Hedman L, Bjerg A, Sundberg S, et al. Both environmental tobacco smoke and personal smoking are related to asthma and wheeze in teenagers. Thorax 2011;66:20-5.

8. Shah S, Peat JK, Mazurski EJ, et al. Effect of peer led programme for asthma education in adolescents: cluster randomised controlled trial. BMJ 2001;322:583-5 